\title{
On the constant factor in Vinogradov's Mean Value Theorem
}

\author{
by
}

\section{GÉzA Kós (Budapest)}

1. Introduction. Vinogradov's Mean Value Theorem gives an upper bound for the integral

$$
J_{k}(b, Q)=\int_{0}^{1} \ldots \int_{0}^{1}\left|\sum_{x=1}^{Q} e\left(\alpha_{1} x+\alpha_{2} x^{2}+\ldots+\alpha_{k} x^{k}\right)\right|^{2 b} d \alpha_{1} \ldots d \alpha_{k}
$$

for positive integers $k, b$ and $Q$. The theorem has many applications in estimating Weyl sums and in several problems of number theory, such as Waring's problem and estimating the value of the Riemann zeta function.

The integral $J_{k}(b, Q)$ has a particular interpretation: it is the number of solutions of the system

$$
\begin{aligned}
x_{1}+x_{2}+\ldots+x_{b} & =x_{b+1}+x_{b+2}+\ldots+x_{2 b}, \\
x_{1}^{2}+x_{2}^{2}+\ldots+x_{b}^{2} & =x_{b+1}^{2}+x_{b+2}^{2}+\ldots+x_{2 b}^{2}, \\
& \vdots \\
x_{1}^{k}+x_{2}^{k}+\ldots+x_{b}^{k} & =x_{b+1}^{k}+x_{b+2}^{k}+\ldots+x_{2 b}^{k},
\end{aligned}
$$

where the variables $x_{1}, \ldots, x_{2 b}$ are from the set $\{1,2, \ldots, Q\}$.

Vinogradov's idea was to estimate Weyl sums using the "average" of all Weyl sums $J_{k}(b, Q)$, and estimate $J_{k}(b, Q)$ as the number of solutions of (2).

Both Vinogradov's theorem and its improvements have the form

$$
J_{k}(b, Q)<C_{k, b} Q^{2 b-k(k+1) / 2+\delta_{k, b}}
$$

where the magnitude order of $\delta_{k, b}$ is $\frac{1}{2} k^{2} \exp \left(-c b / k^{2}\right)$ and the difference is only in the constants. (See for example [2] and [3].) The magnitude order of $C_{k, b}$ is roughly $\exp \left(c k^{3+\varepsilon}\right)$.

Some lower bounds for $J_{k}(b, Q)$ have been done by estimating the integrand near the origin. The goal of these considerations was to determine the

2000 Mathematics Subject Classification: Primary 11L07. 
correct exponent of $Q$, and the results were written as

$$
J_{k}(b, Q)>c_{k, b} Q^{2 b-k(k+1) / 2}
$$

where $c_{k, b} \ll 1 / b^{k}$.

Hua [1] also proved an asymptotics

$$
J_{k}(b, Q)=(1+o(1)) d_{k, b} Q^{2 b-k(k+1) / 2}
$$

for fixed $b>(3+o(1)) k^{2} \log k$ and $Q \rightarrow \infty$. Recently, Wooley [4] proved the same for $b>(1+o(1)) k^{2} \log k$ as well.

In this paper we show that the constant $c_{k, b}$ in (4) can be increased to roughly $k^{c k^{2}} / b^{k}$.

2. The new lower bound. The main result is the following theorem.

THEOREM 2.1. We have

$$
J_{k}(b, Q)>\frac{1 ! \cdot 2 ! \cdot \ldots \cdot k !}{(b+1)^{k}} Q^{2 b-k(k+1) / 2} .
$$

Proof. The idea is to use binomial coefficients instead of powers. The system

$$
\begin{aligned}
\left(\begin{array}{c}
x_{1} \\
1
\end{array}\right)+\ldots+\left(\begin{array}{c}
x_{b} \\
1
\end{array}\right) & =\left(\begin{array}{c}
x_{b+1} \\
1
\end{array}\right)+\ldots+\left(\begin{array}{c}
x_{2 b} \\
1
\end{array}\right), \\
\left(\begin{array}{c}
x_{1} \\
2
\end{array}\right)+\ldots+\left(\begin{array}{c}
x_{b} \\
2
\end{array}\right) & =\left(\begin{array}{c}
x_{b+1} \\
2
\end{array}\right)+\ldots+\left(\begin{array}{c}
x_{2 b} \\
2
\end{array}\right), \\
& \vdots \\
\left(\begin{array}{c}
x_{1} \\
k
\end{array}\right)+\ldots+\left(\begin{array}{c}
x_{b} \\
k
\end{array}\right) & =\left(\begin{array}{c}
x_{b+1} \\
k
\end{array}\right)+\ldots+\left(\begin{array}{c}
x_{2 b} \\
k
\end{array}\right)
\end{aligned}
$$

is equivalent to (2), but the terms are smaller.

For arbitrary integers $a_{1}, \ldots, a_{k}$, denote by $\psi\left(a_{1}, \ldots, a_{k}\right)$ the number of solutions of the system

$$
\left(\begin{array}{c}
x_{1} \\
1
\end{array}\right)+\ldots+\left(\begin{array}{c}
x_{b} \\
1
\end{array}\right)=a_{1}, \quad \ldots, \quad\left(\begin{array}{c}
x_{1} \\
k
\end{array}\right)+\ldots+\left(\begin{array}{c}
x_{b} \\
k
\end{array}\right)=a_{k} .
$$

Then

$$
\begin{aligned}
Q^{2 b} & =\left(\sum_{x_{1}=1}^{Q} \ldots \sum_{x_{b}=1}^{Q} 1\right)^{2}=\left(\sum_{a_{1}=0}^{b\left(\begin{array}{l}
Q \\
1
\end{array}\right)} \ldots \sum_{a_{k}=0}^{b\left(\begin{array}{l}
Q \\
k
\end{array}\right)} \psi\left(a_{1}, \ldots, a_{k}\right)\right)^{2} \\
& \leq\left(\sum_{a_{1}=0}^{b\left(\begin{array}{l}
Q \\
1
\end{array}\right)} \ldots \sum_{a_{k}=0}^{b\left(\begin{array}{l}
Q \\
k
\end{array}\right)} 1\right)\left(\sum_{a_{1}=0}^{b\left(\begin{array}{l}
Q \\
1
\end{array}\right)} \ldots \sum_{a_{k}=0}^{b\left(\begin{array}{l}
Q \\
k
\end{array}\right)} \psi^{2}\left(a_{1}, \ldots, a_{k}\right)\right)
\end{aligned}
$$




$$
<\prod_{\nu=1}^{k}\left(b\left(\begin{array}{l}
Q \\
\nu
\end{array}\right)+1\right) \cdot J_{k}(b, Q) \leq \frac{(b+1)^{k}}{1 ! \cdot 2 ! \cdot \ldots \cdot k !} Q^{k(k+1) / 2} J_{k}(b, Q),
$$

and the proof is complete.

Recently the author has found an expression $H_{k}(b, Q)$ such that if $k$ and $Q$ are fixed and $b \rightarrow \infty$, then $J_{k}(b, Q)=(1+o(1)) H_{k}(b, Q)$. Due to the length and complicated nature of the argument, it will appear in a subsequent paper.

\section{References}

[1] L. K. Hua, Additive Theory of Prime Numbers, Amer. Math. Soc., Providence, RI, 1965, Theorem 15.

[2] I. M. Vinogradov, The Method of Trigonometric Sums in Number Theory, Nauka, Moscow, 1986 (in Russian).

[3] T. D. Wooley, On Vinogradov's mean value theorem, Mathematika 40 (1993), 379399.

[4] - Some remarks on Vinogradov's mean value theorem and Tarry's problem, Monatsh. Math. 122 (1996), 265-273, Theorem 3.

Computer and Automation Research Institute

Kende u. 13-17

H-1111 Budapest, Hungary

E-mail: kosgeza@cs.elte.hu 